\title{
Intrinsic Time in WDW Conformal Superspace
}

\author{
Pavlov A.E. ${ }^{1}$, Pervushin V.N. ${ }^{2}$ \\ ${ }^{1}$ Institute of Mechanics and Energetics, Russian State Agrarian University, Moscow, Russia; \\ ${ }^{2}$ Laboratory of Theoretical Physics, Joint Institute for Nuclear Research, Dubna, Russia; \\ E-mail: Pavlov<alexpavlov60@mail.ru>;
}

In General Relativity there are both York's extrinsic time, constructed of the extrinsic curvature tensor and Misner's intrinsic time, built of the spatial metric tensor. In our paper, to extract the intrinsic time, we generalize the Dirac's mapping of transition to conformal variables. As far as the conformal time is a physical observable as coordinate distance, so the intrinsic time is also a physical magnitude. The choice of the intrinsic time leads inevitably to the Conformal gravitation, where conformal metric is postulated to be a metric of physical space. York's conformal transverse-traceless method of Cauchy problem solution in General Relativity looks quite natural in conformal variables. In Friedmann cosmology, the many-fingered intrinsic time in General Relativity has a sense of a global time of the Universe.

Keywords: Misner's intrinsic time, York's extrinsic time, Dirac's conformal variables, many-fingered time, Conformal gravitation.

DOI: $10.18698 / 2309-7604-2015-1-400-408$

\section{Introduction}

The problem of time and the problem of energy inherently associated with it is a key one in the mathematical General Relativity. Nowadays we see enormous progress in observational cosmology. One can state, that cosmology enters into an era of precise science. Therefore, various theoretical approaches to explaining the fundamental problems are checked with the modern cosmological tests. Our position is that the theory of Einstein's gravity without any modifications provides answers to urgent questions; you just have to obtain them. The Dirac's mapping reflects the transition to physical (conformal) variables. In spirit of ideas of Conformal cosmology [1], the conformal metric is a metric of the space, where we live and make observations. The choice of conformal measurement standards allows us to separate the cosmic evolution of the devices of observation from the evolution of cosmic objects. Thus we avoid unpleasant artefact of expanding Universe and the inevitable problem of Big Bang. Werner Heisenberg in Chapter "Quantum Mechanics and a Talk with Einstein (1925 - 1926)" [2] quoted Albert Einstein's statement: “But on principle, it is quite wrong to try founding a theory on observable magnitudes alone. In reality the very opposite happens. It is the theory which decides what we can observe". This conversation between two great scientists about the status of observable magnitudes in the theory (quantum mechanics or General Relativity) remains actual nowadays. 


\section{Many-fingered intrinsic time in Geometrodynamics}

The ADM formalism supposes the spacetime with interval

$$
g_{\mu v}\left(t, x^{1}, x^{2}, x^{3}\right) d x^{\mu} \otimes d x^{v}
$$

is to foliated into a family of space-like surfaces $\Sigma_{t}$, labeled by the time coordinate $t$, and with spatial coordinates on each slice $x^{1}, x^{2}, x^{3}$. The first quadratic form

$$
\gamma_{i k}\left(t, x^{1}, x^{2}, x^{3}\right) d x^{i} \otimes d x^{k}
$$

defines the induced metric on every slice $\Sigma_{t}$. The components of the extrinsic curvature tensor $K_{i j}$ of every slice are constructed out of the second quadratic form of the hypersurface. The Hamiltonian dynamics of General Relativity is built in an infinite-dimensional degenerate phase space of 3-metrics (1) and densities of their momenta $\pi^{i j}\left(x^{1}, x^{2}, x^{3}, t\right)$. The latter are expressed through the tensor of extrinsic curvature

$$
\pi^{i j}:=-\sqrt{\gamma}\left(K^{i j}-K \gamma^{i j}\right),
$$

Where we introduced the following notations

$$
K^{i j}:=\gamma^{i k} \gamma^{j l} K_{k l}, \quad K:=\gamma^{i j} K_{i j}, \quad \gamma:=\operatorname{det}\left\|\gamma_{i j}\right\|, \quad \gamma_{i j} \gamma^{j k}=\delta_{i}^{k} .
$$

Paul Dirac, in searching of dynamical degrees of freedom of gravitational field, introduced conformal field variables [3]

$$
\tilde{\gamma}_{i j}:=\frac{\gamma_{i j}}{\sqrt[3]{\gamma}}, \quad \quad \tilde{\pi}^{i j}:=\sqrt[3]{\gamma}\left(\pi^{i j}-\frac{1}{3} \pi \gamma^{i j}\right)
$$

There are only five independent pairs $\left(\tilde{\gamma}_{i j}, \pi^{i j}\right)$ per space point, because 


$$
\tilde{\gamma}:=\operatorname{det}\left\|\tilde{\gamma}_{i j}\right\|=1, \quad \tilde{\pi}:=\tilde{\gamma}_{i j} \tilde{\pi}^{i j}=0
$$

The remaining sixth pair

$$
D:=-\frac{1}{3} \ln \gamma \quad \pi_{D}:=\pi
$$

is canonically conjugated. The essence of the transformation (3) lies in fact, that the metric $\tilde{\gamma}_{i j}$ is equal to the whole class of conformally equivalent Riemannian three-metrics $\gamma_{i j}$. So, the conformal variables (3) describe dynamics of shape of the hypersurface of constant volume. The extracted canonical pair $\left(D, \pi_{D}\right)(4)$ has the transparent physical sense: an intrinsic time and the Hamiltonian density of gravitational field. Unfortunately, the Dirac's transformations (3), (4) have a limited range of applicability: they can be used in the coordinates with dimensionless metric determinant.

We should suppose that information of time must be contained in the internal geometry (1) and Hamiltonian must be given by the characteristics of external geometry - tensor of extrinsic curvature $K_{i j}$. It is in agreement with concepts of quantum Geometrodynamics of Bryce DeWitt [4] and John Wheeler [5]. They assumed the cosmological time to be identical the cosmological scale factor. To overcome the difficulties, we use a fruitful idea of bimetric formalism [6]. Spacetime bi-metric theories were founded using some auxiliary background non-dynamical constant metric with coordinate components $f_{i j}(x)$ of some 3-space, Lie dragged along the coordinate time evolution vector. The restriction to only flat background metric is not strong, so there is a possibility of its choosing. To use an auxiliary metric for a generic case of spatial manifold with arbitrary topology, let us take a local tangent space $T\left(\Sigma_{t}\right)_{x}$ as a background space for every local region of our manifold $\Sigma_{t}$.

Let us introduce the scaled Dirac's conformal variables by the following way:

$$
\tilde{\gamma}_{i j}:=\frac{\gamma_{i j}}{\sqrt[3]{\gamma / f}} \quad \quad \tilde{\pi}^{i j}:=\sqrt[3]{\gamma / f}\left(\pi^{i j}-\frac{1}{3} \pi \gamma^{i j}\right)
$$


Here additionally to the determinant $\gamma$ defined in (2), the determinant of background metric $f$ is appeared:

$$
f:=\operatorname{det}\left\|f_{i j}\right\|
$$

The conformal metric $\tilde{\gamma}_{i j}$ (5) is a tensor field, it transforms according to the tensor representation of the group of diffeomorphisms. The scaling variable $(\gamma / f)$ is a scalar field; it is an invariant relative to diffeomorphisms. We add to the conformal variables (5) a canonical pair: a local intrinsic time $D$ and a Hamiltonian density $\pi_{D}$ by the following way

$$
D:=-\frac{1}{6} \ln \left(\frac{\gamma}{f}\right) \quad \pi_{D}:=2 \pi
$$

The formulae (5), (6) define the scaled Dirac's mapping

$$
\left(\gamma_{i j}, \pi^{i j}\right) \mapsto\left(D, \pi_{D} ; \tilde{\gamma}_{i j}, \tilde{\pi}^{i j}\right)
$$

\section{Cauchy problem in Conformal gravitation}

Let us consider the solution of the Cauchy problem following to York [7] in conformal variables. By this way we get components of metric $\tilde{\gamma}_{i j}$ on some hypersurface with a conformal factor as a function of coordinates

$$
\gamma_{i j}:=e^{-2 D} \tilde{\gamma}_{i j}
$$

The matter characteristics - components of energy-momentum tensor in Euler observer reference under the conformal transformation (8) are transforming according to their conformal weights

$$
\tilde{T}_{\perp \perp}:=e^{-4 D} T_{\perp \perp}, \quad\left(\tilde{T}_{\perp}\right)^{i}:=e^{-5 D}\left(\tilde{T}_{\perp}\right)^{i}
$$


The Hamiltonian constraint in the new extended phase space

$$
\tilde{\Delta} e^{-D / 2}-\frac{1}{8} \tilde{\operatorname{R}} e^{-D / 2}+\frac{1}{8 f} \tilde{\pi}_{i j} \tilde{\pi}^{i j} e^{7 D / 2}+\frac{1}{8} \tilde{T}_{\perp \perp} e^{3 D / 2}-\frac{1}{192 f} \pi_{D}^{2} e^{7 D / 2}=0
$$

is called the Lichnerowicz - York equation. Here $\widetilde{\Delta}$ is the conformal Laplacian, $\widetilde{R}$ is the conformal Ricci scalar. The ADM functional of action takes the form

$$
W=\int_{t_{I}}^{t_{0}} d t \int_{\Sigma_{t}} d^{3} X\left[\left(\tilde{\pi}_{L}^{i j}+\tilde{\pi}_{T T}^{i j}\right) \frac{d}{d t} \tilde{\gamma}_{i j}-\pi_{D} \frac{d}{d t} D-N H_{\perp}-N^{i} H_{i}\right]
$$

where the conformal momentum densities are decomposed on longitudinal and traceless transverse parts

$$
\tilde{\pi}^{i j}:=\tilde{\pi}_{L}^{i j}+\tilde{\pi}_{T T}^{i j}
$$

$\boldsymbol{H}_{\perp}$ is the Hamiltonian constraint, $H_{i}$ are momentum constraints, $N, N^{i}$ are Lagrange multipliers.

Substituting the expressed $\pi_{D}(9)$ into the ADM functional (10), we get the functional 1-form with local time $D$

$$
\omega^{1}=\int_{\Sigma_{D}} d^{3} X\left[\left(\tilde{\pi}_{L}^{i j}+\tilde{\pi}_{T T}^{i j}\right) d \tilde{\gamma}_{i j}-\pi_{D}\left(\tilde{\pi}_{L}^{i j}, \tilde{\pi}_{T T}^{i j}, \tilde{\gamma}_{i j}, D\right) d D\right]
$$

Thus, we have implemented the procedure of dereparameterization [8], passing from the coordinate time $t$ description to the intrinsic time $D$ description.

\section{Global intrinsic time in Geometrodynamics}

Set the York's gauge [9] on every slice, which labeled by the coordinate time $t$, 


$$
K \equiv-3 \kappa=K(t)
$$

where $\kappa:=\frac{1}{3}\left(\kappa_{1}+\kappa_{2}+\kappa_{3}\right)$ is a mean curvature of the hypersurface $\Sigma_{t}$ - arithmetic mean of the principal curvatures. One can now introduce an averaging of functions $[10,11]$ for arbitrary manifold. The averaging rate of the function $D$ is defined as an integral over a slice $\Sigma_{t}$ with a volume $V_{0}$

$$
<\frac{d D}{d t}>:=\frac{1}{V_{0}} \int_{\Sigma_{t}} \sqrt{\gamma} \frac{d D}{d t} d^{3} x
$$

Then, one yields the conjugated momentum, having a sense of Hamiltonian

$$
p_{<D>}:=4 V_{0} K
$$

Thus, we obtain, taking into account the gauge (12), a global interval of time $\langle d D\rangle$ is one for all observers. In a particular case, when $K=$ const for every hypersurface, the Hamiltonian (14) does not depend on coordinate time; one maximal slice $(K=0)$ exists at the moment of time symmetry. The ADM action takes the form

$$
W=-\int_{t_{I}}^{t_{0}} d t p_{<D>}<\frac{d D}{d t}>+\int_{t_{I}}^{t_{t}} d t \int_{\Sigma_{t}} d^{3} X\left[\tilde{\pi}^{i j} \frac{d}{d t} \tilde{\gamma}_{i j}-N H_{\perp}-N^{i} H_{i}\right]
$$

The Hamiltonian constraint (9) is algebraic of the second order relative to $K$ that is characteristic for relativistic theories. Reducing the theory, we choose a sign plus in front of the Hamiltonian $p_{<D>}$. Let us notice, if the York's time was chosen, one should have to resolve the Hamiltonian constraint with respect to variable $D$, that looks unnatural difficult.

\section{Global intrinsic time in FRW universe}

The observational Universe with high precision is homogeneous and isotropic. The action of the model is 


$$
W=\int_{t_{I}}^{t_{0}} d t\left[p_{D} \frac{d D}{d t}-N H_{\perp}\right]
$$

where

$$
H_{\perp}=-\frac{1}{24 V_{0}} e^{3 D} p_{D}^{2}-\frac{6 k V_{0}}{a_{0}^{2}} e^{-D}+V_{0} e^{-3 D}(\rho+3 p)
$$

is the Hamiltonian constraint as in classical mechanics. Here $D(t)$ is a generalized coordinate, and $p_{D}$ is its canonically conjugated momentum. Here $V_{0}$ is a volume of the Universe, $k$ is a sign of curvature of the space, $a_{0}$ is a modern scale, $\rho$ is a density, and $p$ is a pressure.

Resolving the constraint (17), we obtain the Friedmann equation

$$
H^{2} \equiv\left(\frac{d D}{d t}\right)^{2}=\frac{1}{6}\left(\rho_{M}+\rho_{\text {rigid }}+\rho_{\text {rad }}+\rho_{\text {curv }}\right) \text {. }
$$

In the left side of equation we see the Hubble parameter, in the right side - the sum of densities of matter, radiation, term of curvature, $\rho_{\text {rigid }}$ corresponds to density of matter with rigid state equation $p=\rho$. The $C D M$ model considered has not dynamical degrees of freedom. According to the Conformal cosmology interpretation, the Friedmann equation has a following sense: it ties the intrinsic time interval $d D$ with the coordinate time intervaldt. The Hubble diagram demonstrates the existence of the intrinsic time itself in the Universe.

A relative changing of wavelength of an emitted photon corresponds to a relative changing of the scale

$$
Z=\frac{\lambda_{0}-\lambda_{I}}{\lambda_{I}}=\frac{a_{0}-a_{I}}{a_{I}}
$$

where $\lambda_{I}$ is a wavelength of an emitted photon, $\lambda_{0}$ is a wavelength of absorbed photon. The Weyl treatment suggests also a possibility to consider 


$$
1+z=\frac{m_{0}}{\left[a(t) m_{0}\right]},
$$

where $m_{0}$ is an atom modern mass. Masses of elementary particles according to Conformal cosmology interpretation becomes running

$$
m(t)=m_{0} a(t)
$$

Instead of an expansion of the Universe (Standard cosmology) we accept the rate of mass (Conformal cosmology). Thus we avoid an unpleasant unresolved problem of initial singularity (Big Bang) in the framework of the Standard cosmology. It is in agreement with the stationary Einstein's conception of the Universe.

\section{References}

1. Pervushin V.N., Pavlov A.E. (2014). Principles of Quantum Universe. Lambert Academic Publishing.

2. Heisenberg W. (1972). Physics and Beyond: Encounters and Conversations. Harper and Raw.

3. Dirac P.A.M. (1959). Fixation of coordinates in the Hamiltonian theory of gravitation. Phys. Rev., Vol. 114, 924.

4. DeWitt B.S. (1967). Quantum theory of gravity. I. The canonical theory. Phys. Rev., Vol. 160, 1113.

5. Wheeler J.A. (1968). Superspace and the nature of quantum geometrodynamics. Battelle Rencontres: 1967 Lectures in Mathematics and Physics, 242.

6. Rosen N. (1940). General relativity and flat space I. Phys. Rev, Vol. 57. P. 147.

7. York J.W. (1979). Kinematics and dynamics of general relativity. Sources of Gravitational Radiation, Cambridge: Cambridge University Press, 83.

8. Wald R.M. (1984). General Relativity. Chicago: The University of Chicago Press.

9. York J.W. (1972). Role of three-geometry in the dynamics of gravitation. Phys. Rev. Lett., Vol. 28,1082 . 
Proceedings of International Conference PIRT-2015

10. Barbashov B.M., Pervushin V.N., Proskurin D.V. (2006). Hamiltonian General Relativity in finite space and cosmological potential perturbations. Int. J. Mod. Phys., Vol. A 21, 5957.

11. Arbuzov A.B., Barbashov B.M., Nazmitdinov R.G., Pervushin V.N., Borowiec A., Pichugin K.N., Zakharov A.F. (2010). Conformal Hamiltonian dynamics of general relativity. Phys. Lett., Vol. B 691, 230. 